\title{
TG/DSC/FTIR characterization of linear geranyl diesters
}

\author{
Marta Worzakowska $\cdot$ Piotr Ścigalski
}

Received: 2 October 2012/ Accepted: 3 December 2012/Published online: 23 December 2012

(C) The Author(s) 2012. This article is published with open access at Springerlink.com

\begin{abstract}
The synthesis, thermal behavior, and characterization of the decomposition products of linear geranyl diesters: digeranyl succinate, digeranyl glutarate, digeranyl adipinate, and digeranyl sebacinate were presented. The linear geranyl diesters were prepared in direct esterification process of a molar stoichiometric ratio of geraniol and suitable acidic reagent in solvent-free medium at $130{ }^{\circ} \mathrm{C}$ using butylstannoic acid as a catalyst. Their structure was confirmed based on FTIR, ${ }^{1} \mathrm{H}$ - and ${ }^{13} \mathrm{C}-\mathrm{NMR}$ spectra. It was proved that the use of tin catalyst allowed decreasing the reaction time and increasing the final conversion of substrates when compared to non-catalyzed process. It considerably simplifies the processing by reduction of the preparation cost and thus this new method of synthesis of aroma diesters may be attractive for practical applications. The thermal behavior of prepared compounds was studied by TG/DSC/FTIR coupled method. TG analysis showed that diesters are thermally stable up to temperatures above $200{ }^{\circ} \mathrm{C}$. The DTG curves confirmed that these decomposition run as a single-stage process. The $T_{\max 1}$ were in the range of $294.5-313.8{ }^{\circ} \mathrm{C}$ depending on the aliphatic chain length $\left(-\mathrm{CH}_{2}-\right)_{\mathrm{n}}$ in the structure of aroma diesters, which was in accordance with DSC data. The analysis of the gases evolved during heating of diesters in inert atmosphere indicated on the asymmetrical disrupt of their bonds. The cleavage of ester bond and O-geranyl bond was expected. It resulted in production of the mixture of derivatives of geraniol (acyclic and alicyclic monoterpene hydrocarbons) like myrcene, ocimene, or limonene as main decomposition products. In addition, the formation of anhydride, lactone,
\end{abstract}

M. Worzakowska $(\bowtie) \cdot$ P. Ścigalski

Department of Polymer Chemistry, Maria Curie-Skłodowska

University, Gliniana 33 Street, 20-614 Lublin, Poland

e-mail: marta.worzakowska@ poczta.umcs.lublin.pl or ketone functionalities among the degradation products clearly confirmed the proposed degradation path of studied diesters.

Keywords Linear geranyl diesters · TG/DSC/FTIR coupled method . Thermal degradation

\section{Introduction}

Terpene esters especially short chain esters, long chain fatty acid esters, and wax esters are commercially one of the most important aroma compounds. They are also among important flavors and fragrance components due to their organoleptic properties [1-5].

They find a wide application in food, beverages, cosmetic, and pharmaceutical products as a component of many dezodorans, domestic and household products, cosmetic formulations, care products, food stuffs, tobaccos, etc. [6-8].

Among acyclic terpene alcohols, the geranyl esters: formates, acetates, propionates, isobutyrates, isovalerates, laureates, etc., are in high demand. These esters are traditionally obtained by extraction from plant materials, microbial fermentation, and enzymatic or chemical method. However, extraction from plant sources, microbial fermentation, and enzymatic methods exhibit a low yield of the desired product and thus impose high processing cost for commercial production [9-16]. So, esters of acyclic terpene alcohols are nearly always made synthetically.

One of the groups of aroma esters is slowly hydrolyzable diesters of non-allylic and allylic perfume alcohols which are useful and important commercial products especially as pro-fragrances ingredients for cleaning and laundry compositions and fabric softener formulations. 
They are always synthesized chemically during non-catalyzed or acid-catalyzed esterification process of suitable alcohol and acid anhydride using strong acid catalyst, e.g., $p$-toluenesulfonic acid, sulfuric acid or hydrochloric acid or amine-catalyzed esterification of alcohol, and acid chloride $[15,16]$. However, the use of strong acid catalysts for the preparation of diesters of allylic alcohols leads to structural rearrangements of substrates like isomerization of allylic alcohol and formation of undesirable by-products, e.g., myrcene. Thus, it results in reduced yields of pure product and connects with necessity of the use of considerable excess of allylic alcohol in esterification process [15, 16]. In contrast, non-catalyzed esterification reaction of allylic alcohols with acid anhydrides progress slowly and often requires excess of alcoholic substrate and higher temperatures in order to obtain high yields of the final product.

As far as we are aware, no information is available about the usage of tin compounds as a catalyst to chemical synthesis of aroma esters as well as their thermal behavior. In this study, butylstannoic acid was utilized as a catalyst instead of strong acid catalysts for preparation of geranyl diesters during direct esterification process of a molar stoichiometric ratio of terpene alcohol and acid anhydrides or dicarboxylic acids in solvent-free medium at mild conditions. In addition, the thermal behavior and characterization of the decomposition products of obtained linear geranyl diesters: digeranyl succinate, digeranyl glutarate, digeranyl adipinate, and digeranyl sebacinate were studied by TG/DSC/FTIR coupled method. The diesters can be successfully used as a flavor additives for many goods, e.g., as the additives in high temperature processing. The studies of their thermal behavior and especially the kind of decomposition products during heating are essential. It can provide more scientific information and better characterization of the prepared materials.

\section{Experimental}

\section{Materials}

Geraniol (trans-3,7-dimethyl-2,6-oktadien-1-ol, $97 \%$ ) and glutaric anhydride $(95 \%)$ were obtained from Fluka. Succinic anhydride (99\%), adipic acid (99\%), and sebacic acid $(98 \%)$ were delivered by Merck. Butylstannoic acid (catalyst) was from Arkema Inc., USA. The reagents were used as received.

\section{Synthesis of linear geranyl diesters}

The reaction mixture consisted of geraniol ( $2 \mathrm{~mol})$, selected acid anhydride or dicarboxylic acid $(1 \mathrm{~mol})$ and catalyst $(0.05 \mathrm{wt} \%)$ was placed in glass flask $(50 \mathrm{~mL})$. The reaction mixture was heated up to $130{ }^{\circ} \mathrm{C}$ and stirred under reduced pressure in a thermostatic oil bath. The progress of the esterification process was monitored by determination of the residual acid content and by estimation of ${ }^{1} \mathrm{H}-\mathrm{NMR}$ spectra. Also, a control synthesis with no catalyst was performed under the same conditions. The final conversion of geraniol and carboxylic groups were presented in Table 1. The basic physical properties were gathered in Table 2. The structure of the product was confirmed based on FT IR, ${ }^{1} \mathrm{H}$ - and ${ }^{13} \mathrm{C}$-NMR, spectra. The thermal stability and the course of the decomposition process of linear geranyl diesters were monitored by TG/DSC/FTIR coupled method.

\section{Analytical methods}

Proton nuclear magnetic resonance $\left({ }^{1} \mathrm{H}-\mathrm{NMR}\right)$ spectra were obtained using an NMR Brucker-Avance 300 MSL (Germany) spectrometer at $300 \mathrm{MHz}$ with deuterated chloroform $\left(\mathrm{CDCl}_{3}\right)$ as the solvent. ${ }^{1} \mathrm{H}$-NMR chemical shifts in parts per million (ppm) were reported downfield from $0.00 \mathrm{ppm}$ using tetramethylsilane (TMS) as an internal reference.

Attenuated total reflection (ATR) were recorded using infrared Fourier transform spectroscopy on spectrometer Brucker TENSOR 27, equipped with diamond crystal (Germany). The spectra were recorded in the spectral range of $600-4,000 \mathrm{~cm}^{-1}$ with 16 scans per spectrum at a resolution of $4 \mathrm{~cm}^{-1}$.

${ }^{13} \mathrm{C}-\mathrm{NMR}$ spectra were recorded on a Brucker 300MSL instrument (Germany). Chemical shifts were referred to chloroform serving as an internal standard.

Viscosity was measured by means of rotating spindle rheometer at $25{ }^{\circ} \mathrm{C}$, Brookfield, model DV-III (Germany).

Density was evaluated using a glass pycnometer with capillary fuse Gay/Lussac $(25 \mathrm{~mL})$ at $23{ }^{\circ} \mathrm{C}$.

Refractive index was determined by refractometer Carl Zeis Jena at $23{ }^{\circ} \mathrm{C}$.

Acid number $\left(\mathrm{mgKOH} \mathrm{g}^{-1}\right)$ was evaluated by titration of the sample against potassium hydroxide using phenolphthalein as an indicator and acetone as a solvent.

Thermal analysis was carried out on a STA 449 Jupiter F1, Netzsch (Germany) under the following operational conditions: heating rate of $10{ }^{\circ} \mathrm{C} \mathrm{min}^{-1}$, a dynamic atmosphere of helium $\left(40 \mathrm{~mL} \mathrm{~min}{ }^{-1}\right)$, temperature range of 40-600 ${ }^{\circ} \mathrm{C}$, sample weight $\sim 10 \mathrm{mg}$, sensor thermocouple type $\mathrm{S}$ TG-DSC. As a reference, empty $\mathrm{Al}_{2} \mathrm{O}_{3}$ crucible was used. The temperature of $5,10,20,50 \%$ of mass loss $\left(T_{5 \%}\right.$, $\left.T_{10 \%}, T_{20 \%}, T_{50 \%}\right)$, and the temperature of the maximum rate of mass loss $\left(T_{\max }\right)$ based on TG-DTG curves were determined. The onset $\left(T_{\text {onset }}\right)$, max $\left(T_{\text {peak }}\right)$, and end $\left(T_{\text {end }}\right)$ temperatures and the enthalpy of decomposition $(\Delta H)$ of diesters based on DSC curves were evaluated. The 
Table 1 The final conversion of geraniol and carboxylic groups

\begin{tabular}{lllll}
\hline Diester & Catalyst & Reaction time/h & Conversion of geraniol/\% $^{\mathrm{a}}$ & Conversion of carboxylic groups/\% $^{\mathrm{b}}$ \\
\hline Digeranyl succinate & + & 5 & 98.3 & 97.8 \\
& - & 8 & 94.4 & 87.7 \\
Digeranyl glutarate & + & 35 & 95.6 & 95.0 \\
& - & 9 & 90.8 & 83.0 \\
Digeranyl adipinate & + & 38 & 97.4 & 96.6 \\
& - & 12 & 92.4 & 85.2 \\
Digeranyl sebacinate & + & 40 & 96.2 & 95.5 \\
& - & 90.5 & 81.2
\end{tabular}

Reaction was carried out at $130{ }^{\circ} \mathrm{C}$, catalyst content $0.05 \mathrm{wt} \%$

${ }^{\mathrm{a}}$ Final conversion of geraniol estimated based on ${ }^{1} \mathrm{H}-\mathrm{NMR}$ spectra

${ }^{\mathrm{b}}$ Final conversion of carboxylic groups based on residual acid content, \pm the presence or the absence of catalyst, respectively

Table 2 Basic properties of linear geranyl diesters

\begin{tabular}{lllll}
\hline Properties & Digeranyl succinate & Digeranyl glutarate & Digeranyl adipinate & Digeranyl sebacinate \\
\hline Viscosity $/ \mathrm{mPa} \cdot \mathrm{s}$ & 13.8 & 17.5 & 27.7 & 38.7 \\
Density $/ \mathrm{g} \mathrm{cm}^{-3}$ & 0.9876 & 0.9963 & 0.9870 & 1.0063 \\
Refractive index & 1.4860 & 1.4850 & 1.4840 & 1.4865 \\
\hline
\end{tabular}

identification of a gas evolved during decomposition process was detected and analyzed by FTIR spectrometer TGA 585 Brucker (Germany) coupling on-line to STA instrument. The FTIR spectrometer with IR cell maintained at $200{ }^{\circ} \mathrm{C}$ was connected on-line to STA instrument by Teflon transfer line with diameter of $2 \mathrm{~mm}$ heated to $200{ }^{\circ} \mathrm{C}$. The FTIR spectra were recorded in the spectral range of $650-4,000 \mathrm{~cm}^{-1}$ with 16 scans per spectrum at a resolution of $4 \mathrm{~cm}^{-1}$.

\section{Results and discussion}

Synthesis and characterization of linear geranyl diesters

Linear geranyl diesters were synthesized by direct catalyzed esterification process using a stoichiometric molar ratio of geraniol $(2 \mathrm{~mol})$ and suitable acid anhydride or dicarboxylic acid (1 mol) (Scheme 1). The progress of the reaction was monitored by the determination of residual acid content and by estimation of ${ }^{1} \mathrm{H}-\mathrm{NMR}$ spectra of the samples. The percentage conversion of carboxylic groups into ester was calculated as being equivalent to acid consumed. The percentage conversion of geraniol into ester was evaluated based on ${ }^{1} \mathrm{H}-\mathrm{NMR}$ spectra. The integration values of methylene protons of geraniol $(\delta=4.20 \mathrm{ppm})$ and methylene protons of geranyl esters $(\delta=4.60 \mathrm{ppm})$ were chosen for the calculation of the percentage conversion of terpene alcohol according to the following equation:
$\%$ Ester conversion $=[\mathrm{E} /(\mathrm{A}+\mathrm{E})] \times 100$, where $\mathrm{E}$ and $\mathrm{A}$ represent the results of the integration of methylene protons of ester and geraniol, respectively [17].

The calculated results are presented in Table 1. As it is clearly visible, the use of the tin catalyst allow obtaining product with high purity during shorter time compared to non-catalyzed process. The final conversion of geraniol and carboxylic groups was higher than $95 \%$ after $5-12 \mathrm{~h}$ depending on the aliphatic chain length in geranyl diesters during catalytic reaction. The final conversion of geraniol and carboxylic groups not exceeded 95 and $87 \%$, respectively, and was achieved after $30-40 \mathrm{~h}$ for non-catalyzed process. The results confirmed that the use of tin catalyst allowed increasing the yield of the main product and significantly shorten the reaction time which certainly may be attractive for practical applications.

The obtained diesters are flavor oils insoluble in water but soluble in most of the organic solvents with viscosities in the range of 13.8-38.7 $\mathrm{mPa} \cdot \mathrm{s}$ (Table 2). Their structure was confirmed based on FTIR, ${ }^{1} \mathrm{H}$ - and ${ }^{13} \mathrm{C}$-NMR spectra.

Figure 1 presents the FTIR spectra of geranyl diesters. It shows all the characteristic absorption signals for prepared products. The bands at $650-960$ and $1,671 \mathrm{~cm}^{-1}$ came from deformation vibrations of $\mathrm{C}=\mathrm{C}$ double bonds $(=\mathrm{C}-\mathrm{H}$, $>\mathrm{C}=\mathrm{C}-\mathrm{H}$ ) and stretching vibrations of non-conjugated $\mathrm{C}=\mathrm{C}$ double bonds of diesters, respectively. The absorption bands at 1,154 and $1,308 \mathrm{~cm}^{-1}$ are characteristics for stretching vibrations of $\mathrm{C}-\mathrm{O}$ bonds (esters of aliphatic acids). Also, the stretching vibrations corresponding to carbonyl groups $\mathrm{C}=\mathrm{O}$ at $1,734 \mathrm{~cm}^{-1}$ are clearly indicated. 
Scheme 1 General synthetic procedure for linear geranyl diesters

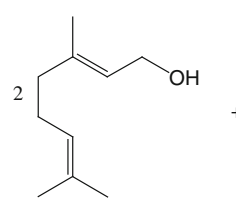

The deformation vibrations of $\mathrm{C}-\mathrm{H}$ at $-\mathrm{CH}_{3}$ groups in the region of $1,357-1,443 \mathrm{~cm}^{-1}$ are visible. The presence of stretching vibrations of $\mathrm{C}-\mathrm{H}$ from $-\mathrm{CH}_{3},-\mathrm{CH}_{2}-$, and $-\mathrm{O}-$ $\mathrm{CH}_{2}-$ groups originate to the double bond in the range of 2,857-2,968 $\mathrm{cm}^{-1}$ are indicated. Moreover, the absence of the characteristic signals for $-\mathrm{OH}$ groups of geraniol at $3,322 \mathrm{~cm}^{-1}$ (Fig. 2) is the confirmation that the esterification process of geraniol and acid component under a catalytic amount of butylstannoic acid was successful and allowed to obtain the desirable product. ${ }^{1} \mathrm{H}-\mathrm{NMR}$ spectra of linear geranyl diesters are shown in Fig. 3. All the protons corresponding to the structure of aroma diesters are observed. The resonance signals attributed to the protons assigned to carbon-carbon double bonds in diester structure: $\delta=5.05-5.15 \mathrm{ppm}$ and $\delta=5.30-5.40 \mathrm{ppm}$ are presence. The resonance signals characteristic for the protons from $-\mathrm{CH}_{2}-$ groups at $2.0-2.15 \mathrm{ppm}$ and the protons from $-\mathrm{CH}_{3}$ groups in geraniol structure in the range of $1.60-1.75 \mathrm{ppm}$ are visible. Also, the protons characteristic for methylene groups $\left(-\mathrm{CH}_{2}-\right)$ in the aliphatic chain of the acidic component of diesters at $2.65 \mathrm{ppm}$ (for digeranyl succinate), at 1.55 and $2.35 \mathrm{ppm}$ (for digeranyl glutarate and digeranyl adipinate), and at $1.25,1.55$ and $2.30 \mathrm{ppm}$ (for digeranyl sebacinate) are clearly visible. The existence of the resonance signals for methylene protons $\left(-\mathrm{CH}_{2}-\right)$ in ester at $\delta=4.60$ and the absence or considerable reduction of the resonance signals for methylene protons in geraniol at $4.2 \mathrm{ppm}$ confirmed the formation of flavor products.

Figure 4 shows the ${ }^{13} \mathrm{C}$-NMR spectra of linear geranyl diesters. The resonance signals for carbon of $-\mathrm{CH}_{3}$ groups at 17,18 , and $27 \mathrm{ppm}$ and of $-\mathrm{CH}_{2}-$ at 26 and $40 \mathrm{ppm}$ and the $\mathrm{C}=\mathrm{C}$ at $124,132,142$, and $119 \mathrm{ppm}$ are observed. Moreover, the presence of the resonance signals for carbon<smiles>CC(C)=CCCC(C)=CCOC(=O)CCC(=O)OCC=C(C)CCC=C(C)C</smiles><smiles>CCCCCC(C)C(=O)OCC=C(C)CCC=C(C)CCC=C(C)C</smiles>

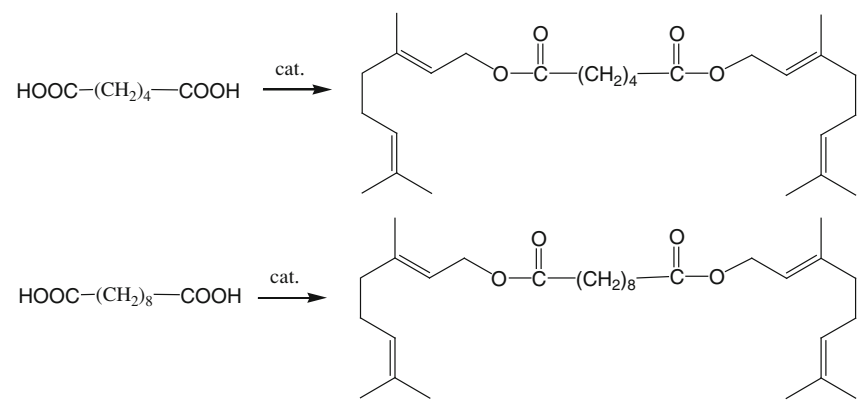

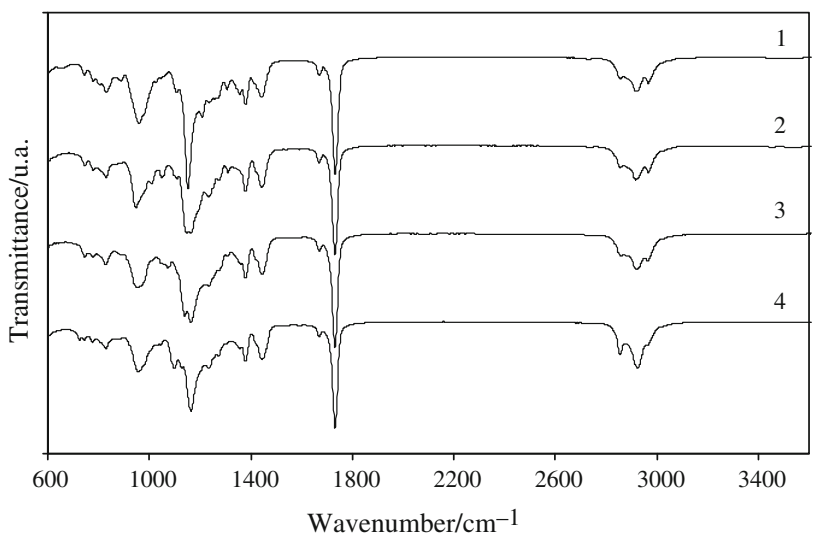

Fig. 1 FTIR spectra of digeranyl succinate (1), digeranyl glutarate (2), digeranyl adipinate (3), and digeranyl sebacinate (4)

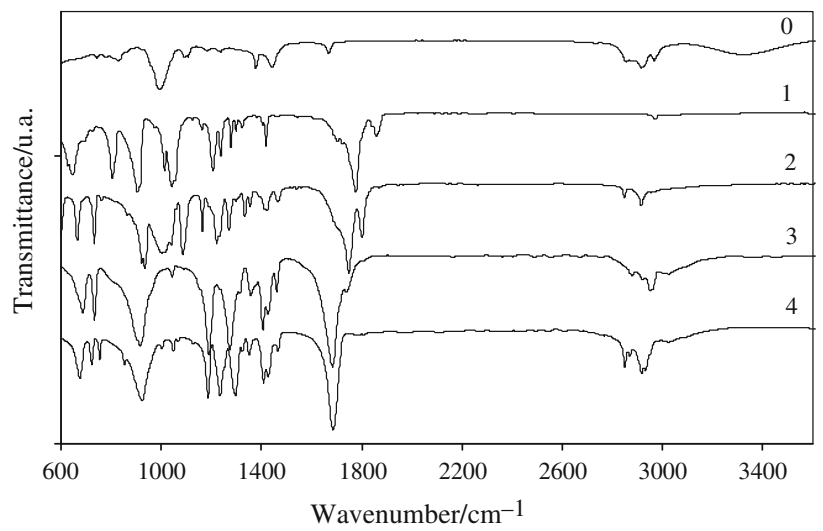

Fig. 2 FTIR spectra of starting materials: geraniol $(0)$, succinic anhydride (1), glutaric anhydride (2), adypic acid (3), and sebacic acid (4) 

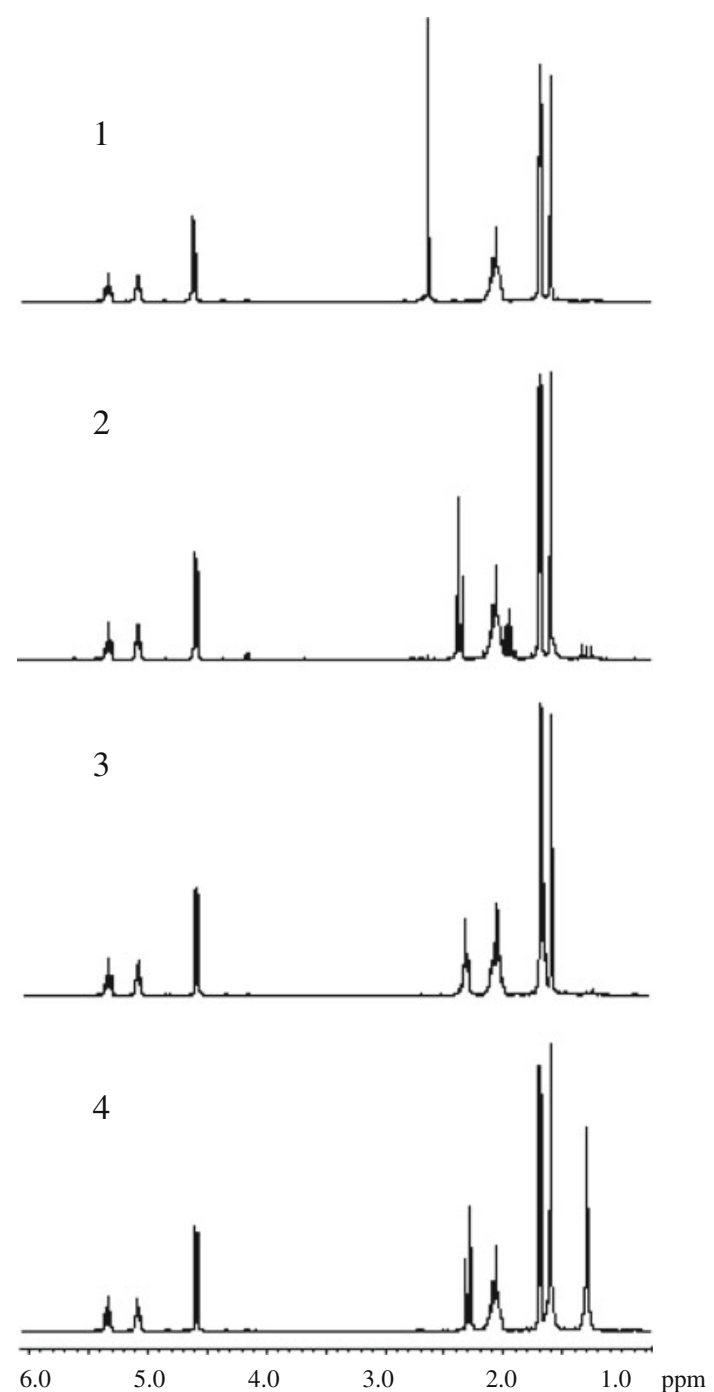

Fig. $3{ }^{1} \mathrm{H}$-NMR spectra of digeranyl succinate (1), digeranyl glutarate (2), digeranyl adipinate (3), and digeranyl sebacinate (4)

from methylene units at $62 \mathrm{ppm}$ and the carbon of $-\mathrm{C}=\mathrm{O}$ at $173 \mathrm{ppm}$ confirmed the formation of ester linkages during preparation process. The carbons from aliphatic chain of acidic component at $29 \mathrm{ppm}$ (digeranyl succinate) 21 and $34 \mathrm{ppm}$ (digeranyl glutarate), at 27 and $35 \mathrm{ppm}$ (digeranyl adipinate), and at 26, 30 and $35 \mathrm{ppm}$ (digeranyl sebacinate) are also indicated.

\section{TG/DSC/FTIR characterization of linear geranyl} diesters

The mass loss curves (TG), the corresponding derivative (DTG), and DSC curves for linear geranyl diesters analyzed in inert atmosphere are presented in Fig. 5. Also, data concerning the results obtained based on those curves are given in Tables 3 and 4 . The diesters are thermally stable up to temperature above $200{ }^{\circ} \mathrm{C}$ in inert atmosphere, except digeranyl glutarate, Table 3 . The lower thermal

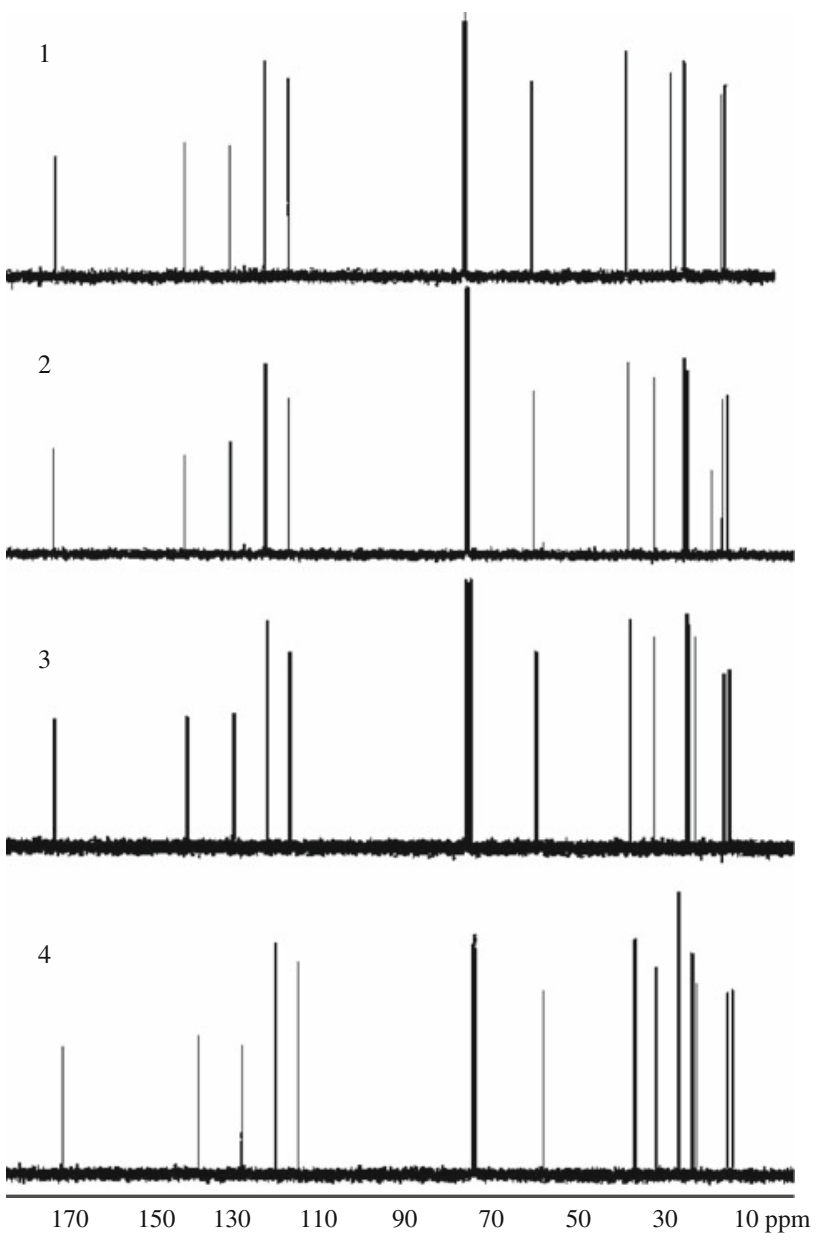

Fig. $4{ }^{13}$ C-NMR spectra of digeranyl succinate (1), digeranyl glutarate (2), digeranyl adipinate (3), and digeranyl sebacinate (4)

stability of digeranyl glutarate was connected with lower conversion of geraniol and glutaric anhydride during synthesis. It resulted in the presence of unreacted substrates in the reaction mixture which was confirmed based on FTIR spectra of evolved gases during heating. The peak at $164{ }^{\circ} \mathrm{C}\left(T_{\max 0}\right)$ connected with their evaporation was observed (Figs. 6, 7). The TG-DTG curves show mass loss in one main step between about 200 and $370{ }^{\circ} \mathrm{C}$ for all studied diesters. In this stage, the mass loss was very fast and significant with the largest mass loss observed at the temperature of the maximum rate of mass loss $\left(T_{\max 1}\right)$ between 294.5 and $313.8{ }^{\circ} \mathrm{C}$ in dependence of the aliphatic chain length $\left(-\mathrm{CH}_{2}-\right)_{n}$ in geranyl diesters. This step is mainly attributed to the total degradation of diesters. Also, the DSC curves clearly show endothermic peak corresponding to the mass loss displayed by the TG curves. $T_{\text {onset }}, T_{\text {peak }}$, and $T_{\text {end }}$ temperatures were comparable to those determined based on DTG curves. The values of enthalpy of decomposition $(\Delta H)$ decreased with increasing aliphatic chain length in diesters from about 408.9 to 
Table 3 TG- DTG data of linear geranyl diesters

\begin{tabular}{|c|c|c|c|c|c|c|c|}
\hline Diester & $T_{5 \%} /{ }^{\circ} \mathrm{C}$ & $T_{10 \%} /{ }^{\circ} \mathrm{C}$ & $T_{20 \%} /{ }^{\circ} \mathrm{C}$ & $T_{50 \%} /{ }^{\circ} \mathrm{C}$ & $T_{\text {onset }} /{ }^{\circ} \mathrm{C}$ & $T_{\text {end }} /{ }^{\circ} \mathrm{C}$ & $T_{\max 1} /{ }^{\circ} \mathrm{C}$ \\
\hline Digeranyl succinate & 230.6 & 249.1 & 265.5 & 285.7 & 264.6 & 306.4 & 294.5 \\
\hline Digeranyl glutarate & 176.1 & 235.5 & 265.7 & 291.2 & 275.4 & 310.5 & $164.0 / 301.4$ \\
\hline Digeranyl adipinate & 235.8 & 248.6 & 260.4 & 291.2 & 276.4 & 311.4 & 301.6 \\
\hline Digeranyl sebacinate & 242.5 & 263.9 & 286.4 & 306.8 & 292.4 & 322.4 & 313.8 \\
\hline
\end{tabular}
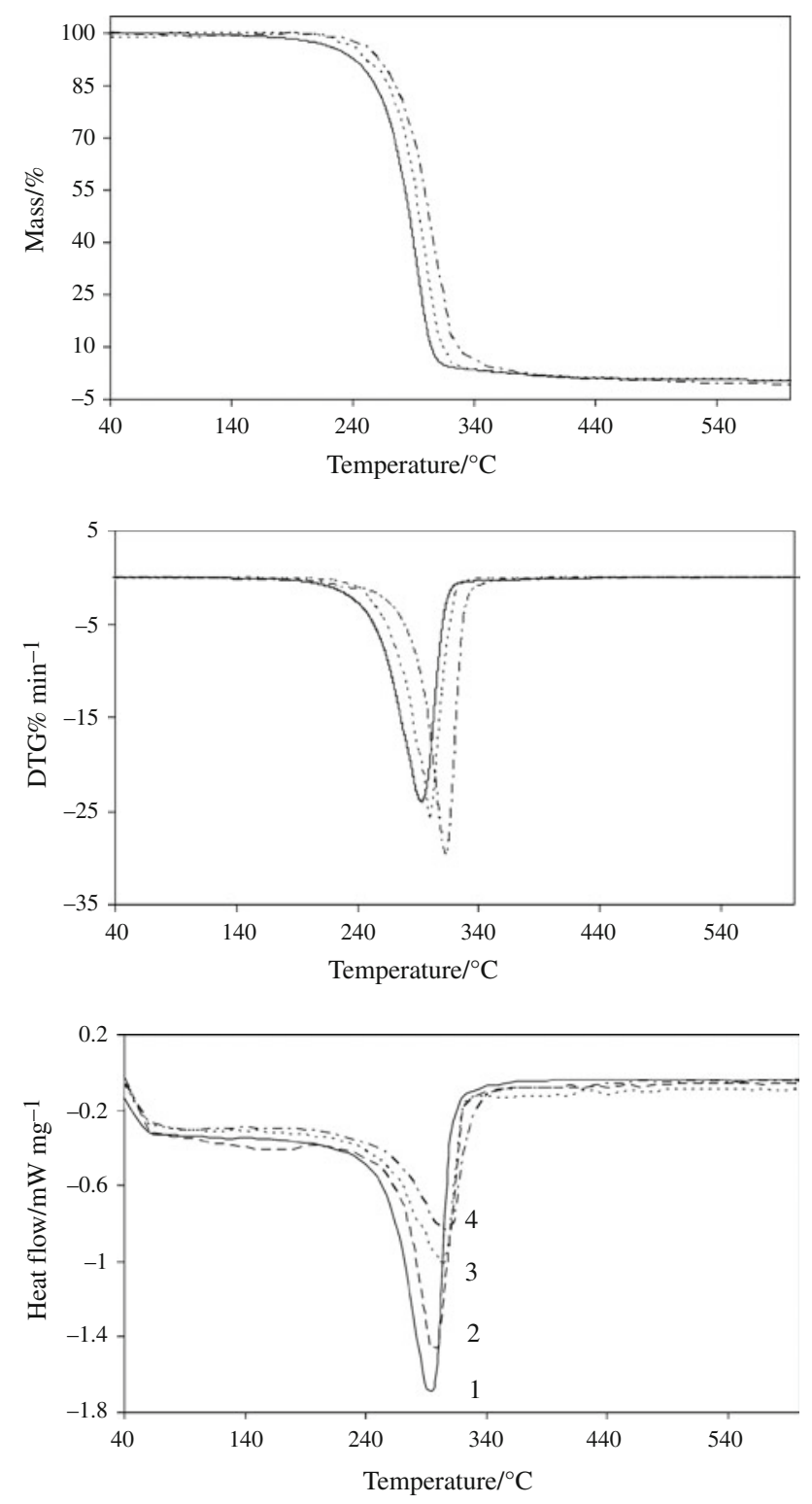

Fig. 5 TG, DTG, and DSC curves for linear geranyl diesters: digeranyl succinate (1), digeranyl glutarate (2), digeranyl adipinate (3), and digeranyl sebacinate (4)

almost $108.9 \mathrm{~J} \mathrm{~g}^{-1}$. It may be connected with the thermal stability of linkages present in the structure of diesters.

Figures 6 and 7 show Gram-Schmidt diagrams and IR spectra of evolved gaseous products during decomposition
Table 4 DSC data of linear geranyl diesters

\begin{tabular}{lllll}
\hline Diester & $T_{\text {onset }} /{ }^{\circ} \mathrm{C}$ & $T_{\text {peak }} /{ }^{\circ} \mathrm{C}$ & $T_{\text {end }} /{ }^{\circ} \mathrm{C}$ & $\Delta H / \mathrm{J} \mathrm{g}^{-1}$ \\
\hline Digeranyl succinate & 259.6 & 294.3 & 311.9 & 408.9 \\
Digeranyl glutarate & 272.1 & $162.3 / 303.2$ & 315.9 & 291.7 \\
Digeranyl adipinate & 275.5 & 302.8 & 318.1 & 154.2 \\
Digeranyl sebacinate & 289.0 & 314.9 & 327.4 & 108.9 \\
\hline
\end{tabular}

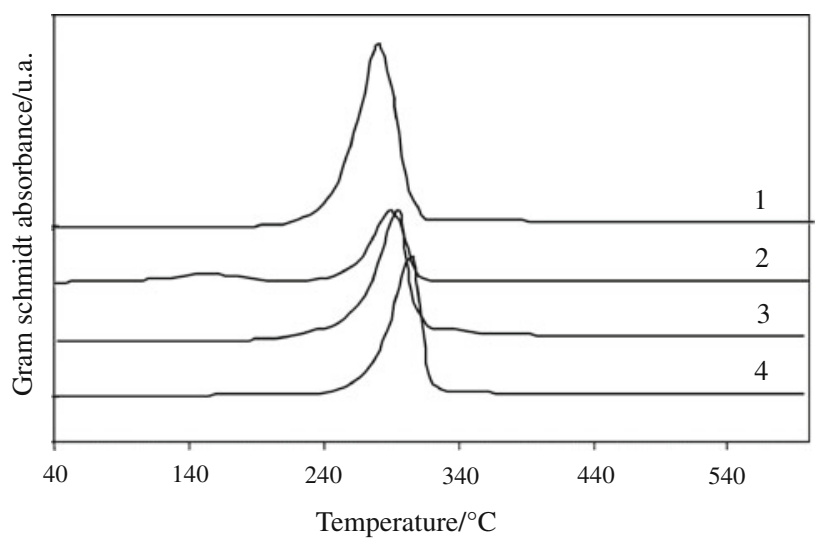

Fig. 6 Gram Schmidt curves for digeranyl succinate (1), digeranyl glutarate (2), digeranyl adipinate (3), and digeranyl sebacinate (4)

of geranyl diesters in inert atmosphere, respectively. The presence of absorbance bands in the range of 2,869-2,977 $\mathrm{cm}^{-1}$ connected with the stretching vibrations of $\mathrm{C}-\mathrm{H}$ for methylene $\left(-\mathrm{CH}_{2}-\right)$ and methyl $\left(-\mathrm{CH}_{3}\right)$ groups originate to the double bond and at $1,382-1,386 \mathrm{~cm}^{-1}$ and $1,449-1,456 \mathrm{~cm}^{-1}$ (the deformation vibrations of $\mathrm{C}-\mathrm{H}$ in methylene groups $\left(-\mathrm{CH}_{3}\right)$ and methyl $\left(-\mathrm{CH}_{2}-\right)$ groups) is observed. Also, the small band at 3,091-3,092 $\mathrm{cm}^{-1}$ was observed. It may indicate on the stretching vibrations of $=\mathrm{C}-\mathrm{H}$ in $-\mathrm{CH}=\mathrm{CH}_{2}$ or $>\mathrm{C}=\mathrm{CH}_{2}$ groups. The out-of-plane deformation vibration of $=\mathrm{C}-\mathrm{H}$ at $828-831 \mathrm{~cm}^{-1}$ for $>\mathrm{C}=\mathrm{CH}-$ groups, at $899-900 \mathrm{~cm}^{-1}$ for $>\mathrm{C}=\mathrm{CH}_{2}$ groups, at $988-990 \mathrm{~cm}^{-1}$ for $-\mathrm{CH}=\mathrm{CH}_{2}$ and trans $-\mathrm{CH}=\mathrm{CH}-$ groups are also visible. In addition, the appearance of stretching vibrations of $\mathrm{C}=\mathrm{C}$ in the region of $1,620-1,680 \mathrm{~cm}^{-1}$ is clearly observed. The occurrence of above absorption bands in IR spectra at $T_{\max 1}$ suggests that the one of the decomposition product of geranyl diesters is acyclic (aliphatic) monoterpene hydrocarbons, e.g., myrcene or 

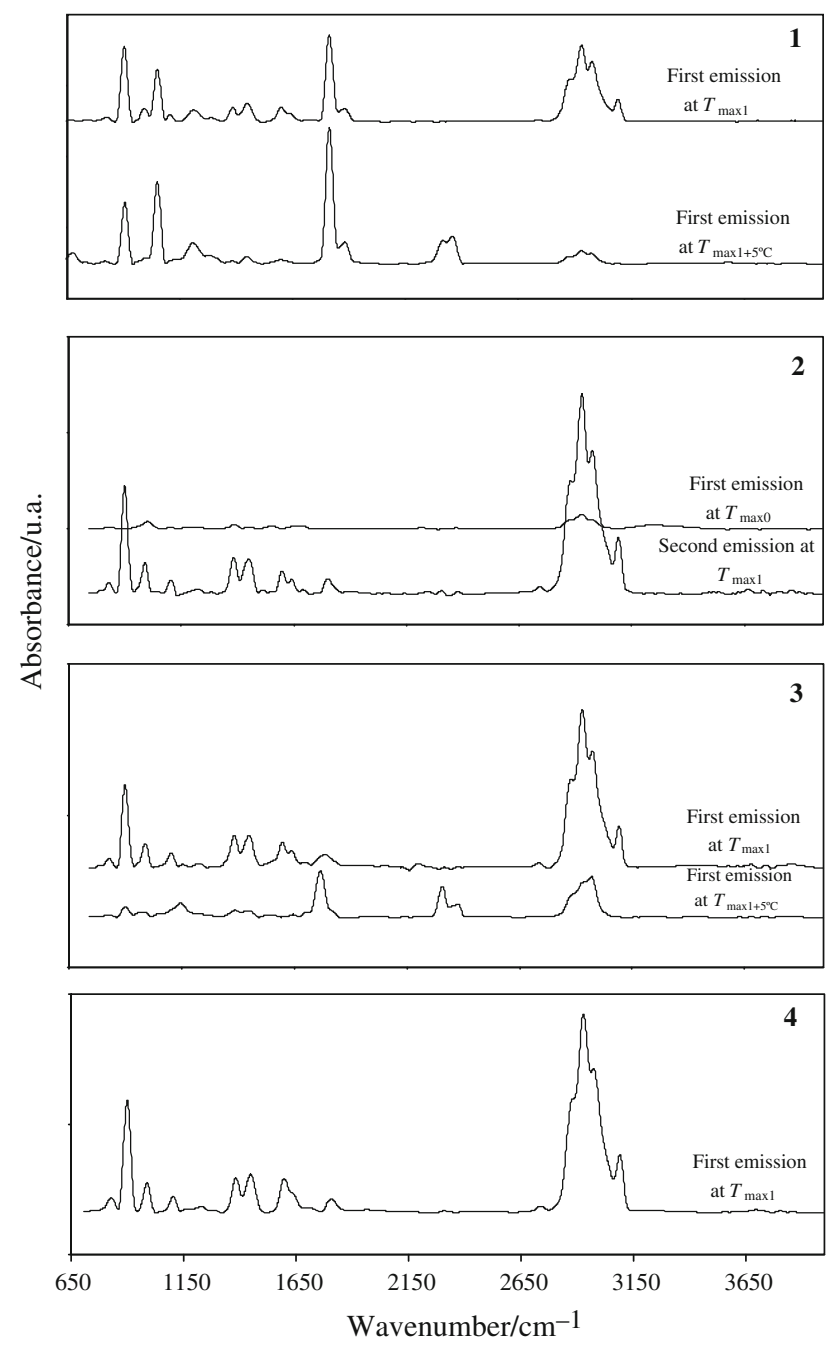

Fig. 7 IR spectra of the gases released during the decomposition of digeranyl succinate (1), digeranyl glutarate (2), digeranyl adipinate (3), and digeranyl sebacinate (4)

trans-ocimene or alicyclic terpene hydrocarbon (limonene) [18]. However, the formation of decomposition products of derivatives of geraniol like isoprene, 1,3-butadiene, or 4-methyl-1,3-pentadiene according to the models proposed in Refs. [19-21] is rather not expected at $T_{\max 1}$. The formation of these decomposition products was observed at relatively higher temperatures according to literature data $[22,23]$.

The appearance of absorption signals at 1,810 with a shoulder at $1,879 \mathrm{~cm}^{-1}$ (stretching vibrations of $\mathrm{C}=\mathrm{O}$ ), 900 and $1,051 \mathrm{~cm}^{-1}$ (stretching vibrations of $\mathrm{C}-\mathrm{O}-\mathrm{C}$ bond) in IR spectra of digeranyl succinate (1) suggests the creating of a five-membered anhydride type ring during the decomposition of ester formed by intramolecular cyclization and its evaporation without further degradation [24]. However, the appearance of the bands in the region of $1,104-1,208 \mathrm{~cm}^{-1}$ (stretching vibration of $\mathrm{C}-\mathrm{O}$ ) might also suggests the formation of etheric or $\beta$-lactone groups.
The IR spectra gathered at $T_{\max }$ of digeranyl glutarate (2) and digeranyl sebacinate (4) (Fig. 7) show the bands at $1,793,1,745$, and $1,045 \mathrm{~cm}^{-1}$ which are connected with the stretching vibrations of $\mathrm{C}=\mathrm{O}$ and $\mathrm{C}-\mathrm{O}$ bands. It suggested the formation of a six-membered anhydride during the decomposition of diester and the evaporation of released acid anhydride. The presence of additional band at $1,162 \mathrm{~cm}^{-1}$ and the bands at 1,793 and $1,045 \mathrm{~cm}^{-1}$ also indicate on the formation of lactone groups (saturated $\gamma$-lactones) during decomposition of those diesters.

The presence of a single absorption band at $1,765 \mathrm{~cm}^{-1}$ was clearly observed from IR spectra of decomposition products of digeranyl adipinate (3), Fig. 7. It could be related to $\gamma$-lactone or acid anhydride groups. The bands at 1,146 and $1,050 \mathrm{~cm}^{-1}$ seem to confirm the predominant presence of these groups. However, the formation of ketone structure is also possible. The small peak in the carbonyl region at $1,701 \mathrm{~cm}^{-1}$ (stretching vibrations of $\mathrm{C}=\mathrm{O}$ groups) could confirm the presence of non-cyclic ketone structure among the decomposition products.

In addition, based on IR spectra of digeranyl succinate (1) and digeranyl adipinate (3), Fig. 7 (first emission at $T_{\max 1+5^{\circ} \mathrm{C}}$ ), the appearance of the absorption bands at 2,329 and 2,358 $\mathrm{cm}^{-1}$ which are attributed to $\mathrm{CO}_{2}$ [25-27] may indicate on the partial decarboxylation process of initially formed products, e.g., acid anhydrides at higher temperatures.

\section{Conclusions}

The linear geranyl diesters were prepared in a direct esterification process of a stoichiometric ratio of geraniol and suitable acidic component in solvent-free medium at $130{ }^{\circ} \mathrm{C}$ using butylstannoic acid as a catalyst. It was proved that use of tin catalyst allowed decreasing the reaction time and increasing the final conversion of substrates considerably when compared to non-catalyzed process. It was confirmed based on determination of the residual acid content and by estimation of ${ }^{1} \mathrm{H}-\mathrm{NMR}$ spectra. It considerably simplifies the processing by reduction of the preparation cost and thus this new method of synthesis of aroma diesters may be attractive for practical applications. The structure of geranyl diesters was analyzed based on FTIR, ${ }^{1} \mathrm{H}$ - and ${ }^{13} \mathrm{C}$-NMR. The thermal degradation of prepared compounds has been assessed using TG/DSC/FTIR coupled method. It was found that the thermal degradation pattern of these compounds was comparable and run as a single-stage process. The diesters were thermally stable up to $200{ }^{\circ} \mathrm{C}$. The $T_{\max 1}$ were in the range of $294.5-313.8{ }^{\circ} \mathrm{C}$ and depended on the aliphatic chain length in their structure. The characterization of gases evolved during heating indicated on asymmetrical disrupt of the bonds in the 
structure of diesters. The cleavage of ester and O-geranyl bonds was expected. The main degradation products are derivatives of geraniol: acyclic and alicyclic terpenes hydrocarbons. The identification of anhydride, lactone, and ketone functionalities among the degradation products of diesters clearly confirmed the degradation path of prepared flavor compounds.

Open Access This article is distributed under the terms of the Creative Commons Attribution License which permits any use, distribution, and reproduction in any medium, provided the original author(s) and the source are credited.

\section{References}

1. Misra S, Ghosh A. Analysis of epicuticular waxes. In: Linskens HF, Jackson JF, editors. Essential oils and waxes. New York: Springer; 1992. p. 205-29.

2. Keng PS, Basri M, Zakaria MRS, Abdul Rahman MB, Ariff AB, Abdul Rahman RNZ, Salleh AB. Newly synthesized palm esters for cosmetic industry. Ind Crop Prod. 2009;29:37-44.

3. Peter TR, Robert B. Beeswax through the ages. Personal Care. 2001;10:27-31.

4. Habulin M, Šabeder S, Paljevac M, Primožič M, Knez Ž. Lipasecatalyzed esterification of citronellol with lauric acid in supercritical carbon dioxide/co-solvent media. J Supercrit Fluid. 2003;43:199-203.

5. Chatterjee T, Bhattacharyya DK. Synthesis of terpene esters by an immobilized lipase in a solvent-free system. Biotechnol Lett. 1998;20:865-8.

6. Chen W, Viljoen AM. Geraniol-a review of a commercially important fragrance material. S Afr J Bot. 2010;76:643-51.

7. Gautschi M, Bajgrowicz JA, Kraft P. Fragrance chemistrymilestones and perspectives. Chimia. 2001;55:379-87.

8. Bedoukian PZ. Geraniol and Nerol. Perfumery and flavoring synthetics. Wheaton: Allured Publishing Corporation; 1986. p. 173-81.

9. Bauer K, Garbe D, Surburg H. Common fragrance and flavor materials: preparation, properties and uses. New York: Wiley; 2001. p. 43-5.

10. Schindler J, Schmid RD. Fragrance or aroma chemicals-microbial synthesis and enzymatic transformation-a review. Process Biochem. 1982;17:2-8.

11. Croteau R. Fragrance and flavour substances. Germany: D\&PS Verlag; 1980. p. 13-4
12. Welsh WW, Murray WD, Williams RE. Microbiological and enzymatic production of flavor and fragrance chemicals. Crit Rev Biotechnol. 1989;9:105-69.

13. Stamatis H, Christakopoulos P, Kekos D, Macris BJ, Kolisis FN. Studies on the synthesis of short-chain geranyl esters catalyzed by Fusarium oxysporum esterase in organic-solvents. J Mol Catal B. 1998;4:229-36.

14. Athawale V, Manjrekar N, Athawale M. Lipase-catalysed synthesis of geranyl methacrylate by transesterification: study of reaction parameters. Tetrahedron Lett. 2002;43:4797-800.

15. Gildemeister E, Hoffmann FR. The volatile oils. New York: Wiley; 1913.

16. Armstrong DW, Gillies B, Yamazaki H. Natural flavors produced by biotechnological processing. In: Charalambous $\mathrm{G}$, editor. Flavor chemistry, trends and development. New York: Elsevier; 1989. p. 104-20.

17. Patil D, Das D, Nag A. Enzymatic synthesis and analytical monitoring of terpene ester by ${ }^{1} \mathrm{H}$ NMR spectroscopy. Chem Pap. 2011;65:9-15.

18. Xie WC, Tan ZC, Gu XH, Tang J, Wang GY, Luo CR, Sun LX. Thermal decomposition of two synthetic glycosides by TG, DSC and simultaneous Py-GC-MS analysis. J Therm Anal Calorim. 2007;87:505-10.

19. Erman WE. In: Gassman PG, editor. Chemistry of the monoterpenes, Part B, Chap. 10. New York: Marcel Dekker; 1985, p. 929-35.

20. Fengel D, Wegener G. Wood: chemistry, ultrastructure, reactions. Berlin: Walter de Gruyter; 1984. p. 182.

21. Gwyn JE. Universal yield models for the steam pyrolysis of hydrocarbons to olefins. Fuel Process Technol. 2001;70:1-7.

22. Runckel WJ, Goldblatt LA. Inhibition of myrcene polymerization during storage. Ind Eng Chem. 1946;38:749-51.

23. Kolicheski MB, Cocco LC, Mitchell DA, Kaminski M. Synthesis of myrcene by pyrolisis of $\beta$-pinene: analysis of decomposition reactions. J Anal Appl Pyrol. 2007;80:92-100.

24. Caires FJ, Lima LS, Carvalho CT, Ionashiro M. Thermal behaviour of succinic acid, sodium succinate and its compounds with some bivalent transition metal ions. Thermochim Acta. 2010;500:6-12.

25. Ionashiro EY, Caires FJ, Siqueira ABS, Lima LS, Carvalho CT. Thermal behaviour of fumaric acid, sodium fumarate and its compounds with light trivalent lanthanides. J Therm Anal Calorim. 2012;108:1183-8.

26. Tănase C, Odochian L, Apostouscu N, Pui A. TG-FTIR analysis applied to the study of thermal behaviour of some edible mushrooms. J Therm Anal Calorim. 2011;103:1079-85.

27. Shi J, Wang Z, Liu Y, Wang C. Investigation of thermal behavior of enoxacin and its hydrochloride. J Therm Anal Calorim. 2012;108:299-306. 